\title{
A CLASS OF AFFINELY CONNECTED SPACES
}

\author{
BY \\ HSIEN-CHUNG WANG AND KENTARO YANO
}

It is known that the existence of a group $G$ of motions of sufficiently high dimension in an $n$-dimensional Riemannian space imposes strong restrictions on the space $[10 ; 15 ; 16]$. This restriction is related to the fact that the isotropic subgroup, being a "high" dimensional subgroup of the orthogonal group $O(n)$, is very restricted [12]. Some results of this nature for affine connections have been known $[4 ; 5 ; 6 ; 7 ; 8 ; 11 ; 13]$; in particular, if an affinely connected space $\mathfrak{A}_{n}$ of dimension $n$ admits a group $G$ of collineations with $\operatorname{dim} G$ $\geqq n^{2}-n+5$, then $\mathfrak{A}_{n}$ is projectively flat. We know however that, unlike the Riemannian case, there are numerous non-equivalent, simply-connected, homogeneous $\left({ }^{1}\right)$ affinely connected spaces with the same local structure. For example, over the Euclidean space (in the topological sense), many flat and homogeneous affine connections can be defined such that any two of them are different in the global sense $\left({ }^{2}\right)$. This tells us that the above class of spaces $\mathfrak{A}_{n}$ needs some further clarifications.

In this paper, we give a more detailed study of the symmetric affinely connected spaces $\mathfrak{A}_{n}$ having the above property. All the possible curvature tensors of $\mathfrak{A}_{n}$ as well as the isotropic subgroup of $G$ are determined (Theorem $2, \S 9)$. In case $G$ is transitive, we exhibit all the simply-connected $\mathfrak{A}_{n}$ 's (Theorem 3, §10). They fall into three individual cases and two classes depending on a non-negative constant. Among them, there are three affinely flat spaces, two of which are homeomorphic with the euclidean space while the other one homeomorphic with the product of a line and an $(n-1)$-sphere, $n>2$. As for the nonflat cases, they are all homeomorphic with the euclidean space. For the transitive case, all the isotropic subgroups given in Theorem 2 are realized; some of them can moreover be realized over nonequivalent affinely connected spaces.

1. Reducibility of certain linear groups. Let $P_{n}$ denote the special linear group of $n$ real variables $x^{1}, \cdots, x^{n}$. In this section and the next, we shall determine all the closed subgroups of $P_{n}$ with dimension not less than $n^{2}-2 n+4$. This information plays an important rôle in our further discussions. Let us first establish the following

Received by the editors December 28, 1953 and, in revised form, October 16, 1954.

(1) An affinely connected space is called homogeneous if it admits a transitive group of collineations.

(2) Let $\mathfrak{B}_{n}$ be the entire $n$-space with coordinates $x^{1}, x^{2}, \cdots, x^{n}$ and $\mathfrak{B}_{n}(m)$ the subset of $\mathfrak{B}_{n}$ consisting of all the points whose first $m$ coordinates are positive. The affinely connected spaces $\left\{\mathfrak{B}_{n}(m) ; \Gamma^{i} \boldsymbol{i}_{k}(x)=0\right\}(m=0,1,2, \cdots, n)$ are homogeneous, flat, and homeomorphic with one another. But no two of them are equivalent. 
Lemma 1. Let $G$ be a proper subgroup of $P_{n}$. If $\operatorname{dim} G \geqq n^{2}-2 n+4$, then $G$ is reducible.

Proof. We shall prove it by using the method of contradiction. Suppose $G$ to be irreducible. Let $G$ denote the same group $G$ when the range of the variables $x^{1}, \cdots, x^{n}$ is extended to the field of complex numbers. Thus $G$ is a transformation group of the complex vector space of $n$-dimensions. Of course,

$$
\operatorname{dim} G=\operatorname{dim} G \geqq n^{2}-2 \mathrm{n}+4 .
$$

If $G$ is reducible, then as a direct consequence of Cartan's arguments [1, p. $155]$ we would have $\operatorname{dim} G \leqq(n / 2)^{2}$ which is impossible. Thus $G$ is irreducible, and thus the complex form $\Gamma$ of $G$ is also irreducible. We know that $[1$, p. 151] a complex irreducible linear group is either semi-simple or the direct product of a semi-simple group and the homothetic group defined by $y^{i}=\rho x^{i}$ where $\rho$ is an arbitrary nonvanishing complex number. From our assumption $G \subset P_{n}$, no homothetic transformation can appear in $\Gamma$. Hence $\Gamma$ is semi-simple. Here we find it convenient to divide our discussion into the following two cases:

CASE $1 . \Gamma$ is simple. Since the complex dimension of $\Gamma$ which is the same as $\operatorname{dim} G$ is not less than $n^{2}-2 n+4$, we know that $\Gamma$ cannot be isomorphic with a complex linear group in less than $n$ variables. Such simple linear groups have been determined by Cartan. Taking account of the fact $\operatorname{dim} \Gamma$ $\geqq n^{2}-2 n+4$, a survey of Cartan's table [3, pp. 147-148] tells us at once that $\operatorname{dim} \Gamma=n^{2}-1$ where $\operatorname{dim} \Gamma$ means the complex dimension. Hence $\operatorname{dim} G$ $=n^{2}-1$. This contradicts our assumption that $G$ is a proper subgroup of $P_{n}$.

CASE 2. $\Gamma$ is not simple. From representation theory, $\Gamma$ can be written as a Kronecker product $\Gamma_{1} \times \Gamma_{2}$ of two nontrivial irreducible linear groups $\Gamma_{1}$ and $\Gamma_{2}$. Let $r_{i}$ and $n_{i}$ denote, respectively, the complex dimension and degree $\left(^{3}\right)$ of $\Gamma_{i}(i=1,2)$. Then

$$
n_{1} n_{2}=n, \quad r_{1}+r_{2}=\operatorname{dim} \Gamma \geqq n^{2}-2 n+4, \quad n_{1} \geqq 2, \quad n_{2} \geqq 2 .
$$

Without loss of generality, we can assume $r_{1} \geqq\left(n^{2}-2 n+4\right) / 2$. Since $n_{1} \leqq n / 2$, it follows that $r_{1} \geqq n_{1}^{2}-1$. A contradiction is thus obtained.

The contradiction in both alternatives proves our lemma.

2. Subgroups of the affine groups. Let $H_{n}$ be the general linear group in $n$ real variables. Then each element of $H_{n}$ can be regarded as a nonsingular real matrix $\left(a_{i j}\right)$, and the special linear group $P_{n}$ becomes the totality of matrices $\left(a_{i j}\right)$ whose determinants det $\left(a_{i j}\right)$ are equal to one. For simplicity, we shall use the following notations throughout:

$$
\begin{aligned}
H_{n}^{+} & =\left\{\left(a_{i j}\right): \operatorname{det}\left(a_{i j}\right)>0\right\}, \\
K & =\left\{\left(a_{i j}\right): a_{i j}=\lambda \delta_{i j}, \lambda=\text { positive number }\right\}\left({ }^{4}\right),
\end{aligned}
$$
acts.

(3) By the degree of a linear group, we mean the dimension of the vector space on which it

(4) $\delta_{i j}, \delta_{j}^{i}$ denote the Kronecker deltas. 


$$
\begin{aligned}
L & =\left\{\left(a_{i j}\right): a_{11}=1, a_{\alpha 1}=0, \operatorname{det}\left(a_{i j}\right)=1 ; \alpha=2,3, \cdots, n\right\}, \\
L^{\prime} & =\left\{\left(a_{i j}\right): a_{11}=1, a_{1 \alpha}=0, \operatorname{det}\left(a_{i j}\right)=1 ; \alpha=2,3, \cdots, n\right\}, \\
M & =\left\{\left(a_{i j}\right): a_{11}>0, a_{\alpha 1}=0, \operatorname{det}\left(a_{i j}\right)=1 ; \alpha=2,3, \cdots, n\right\}, \\
M^{\prime} & =\left\{\left(a_{i j}\right): a_{11}>0, a_{1 \alpha}=0, \operatorname{det}\left(a_{i j}\right)=1 ; \alpha=2,3, \cdots, n\right\},
\end{aligned}
$$

where, and in the following, the indices $a, b, c, \cdots, i, j, k, \cdots$ take the values in the range $1,2,3, \cdots, n$.

We see at once that they are closed and connected subgroups of $\mathrm{H}_{n}^{+}$, and

$$
\begin{aligned}
\operatorname{dim} H_{n}^{+}=n^{2}, & \operatorname{dim} K=1, \\
\operatorname{dim} L=\operatorname{dim} L^{\prime}=n^{2}-n-1, & \operatorname{dim} M=\operatorname{dim} M^{\prime}=n^{2}-n .
\end{aligned}
$$

Lemma 2. Let $G$ be a closed and connected subgroup of $M$ with $\operatorname{dim} G \geqq n^{2}$ $-2 n+4$. Then either $G=L$, or $G=M$.

Proof. Let

$$
P_{n-1}=\left\{\left(a_{i j}\right): a_{11}=1, a_{1 \alpha}=a_{\alpha 1}=0, \operatorname{det}\left(a_{i j}\right)=1 ; \alpha=2,3, \cdots, n\right\} \text {. }
$$

Since $G, L$, and $P_{n-1}$ are all subgroups of $M$, we have

$$
\begin{aligned}
& \operatorname{dim}\left(G \cap P_{n-1}\right)=\operatorname{dim} G+\operatorname{dim} P_{n-1}-\operatorname{dim} M \geqq n^{2}-3 n+4, \\
& \operatorname{dim}\left(L \cap P_{n-1}\right)=\operatorname{dim} L+\operatorname{dim} P_{n-1}-\operatorname{dim} M \geqq n_{2}-3 n+3 .
\end{aligned}
$$

It is a well known result (due to $\mathrm{S}$. Lie) that the projective group $P_{k}$ has no proper subgroup with dimension higher than $k^{2}-k$. Thus $P_{n-1}$ cannot have proper subgroup with dimension higher than $n^{2}-3 n+2$. (2.1) then implies that $G \cap P_{n-1}=P_{n-1}$, or what is the same, $P_{n-1} \subset G$. Thus $P_{n-1} \subset G \cap L \subset L$. By using matrix multiplication we can easily verify that $P_{n-1}$ is a maximal subgroup of $L$. It follows then that $G \cap L$ is either $P_{n-1}$ or $L$. On account of (2.2), the first alternative cannot happen, and therefore, $G \cap L=L$, i.e., $L \subset G$. But we know that the difference between the dimensions of $L$ and $M$ is equal to one. It follows then that $G$ is either $L$ or $M$. Lemma 2 is proved.

TheOREM 1. Let $G$ be a closed and connected subgroup of $P_{n}$. If $\operatorname{dim} G \geqq n^{2}$ $-2 n+4$, then $G$ is conjugate to one of the groups $P_{n}, L, M, L^{\prime}, M^{\prime}$.

Proof. If $G=P_{n}$, our theorem evidently holds. Now, assume $G \neq P_{n}$. Lemma 1 then tells us that $G$ is reducible. In other words, $G$ leaves invariant a linear subspace of $m$ dimensions with $0<m<n$. Thus $\operatorname{dim} G \leqq n^{2}-1$ $-m(n-m)$. From the inequality $\operatorname{dim} G \geqq n^{2}-2 n+4$, it follows that $m$ is either 1 or $n-1$. These two cases are dual to each other. Suppose that $m=1$. Then $G$ leaves invariant a line. Up to an inner automorphism of $P_{n}$, we can assume this invariant line to be

$$
x^{2}=x^{3}=\cdots=x^{n}=0 .
$$

Thus $G$ is subgroup of $M$. From Lemma 2, it follows that $G$ is either $L$ 
or $M$. By a duality argument, we see that in case $m=n-1, G$ is either $L^{\prime}$ or $M^{\prime}$. Theorem 1 is hereby proved.

Corollary. For each real number $b$, let us denote by $I(b)$ the totality of diagonal matrices of the form:

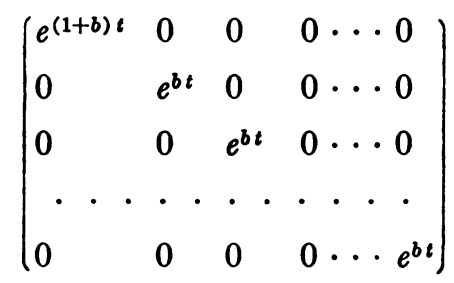

where $t$ runs through all real numbers. Then each closed and connected subgroup $G$ of $H_{n}$ with $\operatorname{dim} G \geqq n^{2}-2 n+5$ is conjugate to one of the groups: $H_{n}^{+}, P_{n}$, $K \times M, K \times M^{\prime}, K \times L, K \times L^{\prime}, I(b) \times L, I(b) \times L^{\prime}, L, L^{\prime}$.

Proof. From the connectedness of $G$, we know $G \subset H_{n}^{+}$. Let $G^{*}=G \cap P_{n}$. Since $H_{n}^{+}=K \times P_{n}$ and $\operatorname{dim} K=1$, it follows that $G^{*}$ is a normal subgroup of $G$ and $\operatorname{dim} G^{*} \leqq \operatorname{dim} G \leqq \operatorname{dim} G^{*}+1$. This tells us that $\operatorname{dim} G^{*} \geqq n^{2}-2 n+4$. Then on account of Theorem 1, we can assume $G^{*}$ to be one of the groups $P_{n}, L, M, L^{\prime}, M^{\prime}$. When $G^{*}=P_{n}, G$ is evidently either $P_{n}$ or $H_{n}^{+}$. We shall only discuss the cases $G^{*}=L$ and $G^{*}=M$; the remaining two cases can be reduced to these two by a duality.

CASE 1. $G^{*}=M$. Since $G^{*}$ is a normal subgroup of $G, G$ is contained in the normalizer $K \times M$ of $M$. From the fact that $M \subset G \subset K \cap M, \operatorname{dim} K=1$, we know that $G$ can only be $M$ or $K \times M$. But $M=I(-1 / n) \times L$. Thus our corollary holds in this case.

CASE 2. $G^{*}=L$. The normalizer of $L$ in $H_{n}^{+}$is $K \times M$. Hence $L \subset G \subset K \times M$. Passing from these groups to their Lie algebras, we find by a short calculation that $G$ is either $L$ or $K \times L$ or $I(b) \times L$ for a certain $b$. This completes the proof of the corollary.

3. Groups of affine collineations. Let $\mathfrak{A}_{n}$ be an $n$-dimensional space with symmetric affine connection $\Gamma_{j k}^{t}(x)$ covered by a system of coordinate neighborhoods $\left(x^{i}\right)$. Then the paths of this space are defined to be integral curves of the differential equations

$$
\frac{d^{2} x^{i}}{d s^{2}}+\Gamma_{j k}^{i} \frac{d x^{i}}{d s} \frac{d x^{k}}{d s}=0
$$

$s$ being the so-called affine parameter on each path. A point transformation

$$
\bar{x}^{i}=f^{i}(x)
$$

is called an affine collineation when it carries any path into a path of the space and preserves the affine character of the parameter $s$. A necessary and suffi- 
cient condition that (3.2) be an affine collineation is that

$$
\Gamma_{j k}^{i}(\bar{x})=\frac{\partial \bar{x}^{i}}{\partial x^{a}}\left(\frac{\partial x^{b}}{\partial \bar{x}^{j}} \frac{\partial x^{c}}{\partial \bar{x}^{k}} \Gamma_{b c}^{a}(x)+\frac{\partial^{2} x^{a}}{\partial \bar{x}^{j} \partial \bar{x}^{k}}\right) .
$$

If (3.2) is an infinitesimal transformation

$$
\bar{x}^{i}=x^{i}+\xi^{i}(x) d t,
$$

$\xi^{i}(x)$ being a vector field and $d t$ an infinitesimal, then (3.3) gives

$$
X \Gamma_{j k}^{i} \equiv \frac{\partial^{2} \xi^{i}}{\partial x^{j} \partial x^{k}}+\xi^{a} \frac{\partial \Gamma_{j k}^{i}}{\partial x^{a}}-\frac{\partial \xi^{i}}{\partial x^{a}} \Gamma_{j k}^{a}+\frac{\partial \xi^{a}}{\partial x^{j}} \Gamma_{a k}^{i}+\frac{\partial \xi^{a}}{\partial x^{k}} \Gamma_{j a}^{i}=0
$$

where $X$ is the operator of Lie derivation with respect to (3.4). Equation (3.5) may be written also in tensor form:

$$
X \Gamma^{i}{ }_{j k} \equiv \xi^{i} ; j ; k+R^{i}{ }_{j k l} \xi^{l}=0,
$$

where the semi-colon followed by an index denotes the covariant differentiation with respect to $\Gamma^{i}{ }_{j k}$ and $R^{i}{ }_{j k l}$ the curvature tensor formed with $\Gamma^{i}{ }_{j k}$.

The integrability conditions of system (3.6) of partial differential equations, or those of

$$
\xi_{j ; k}=-R^{i}{ }_{j k l} \xi^{l}
$$

are given by a sequence of equations

$$
\begin{aligned}
& X R^{i}{ }_{j k l} \equiv \xi^{a} R^{i}{ }_{j k l ; a}-\xi^{i}{ }_{i a} R^{a}{ }_{j k l}+\xi^{a}{ }_{j ;} R^{i}{ }_{a k l}+\xi^{a}{ }_{i k} R^{i}{ }_{j a l}+\xi^{a}{ }_{j l} R^{i}{ }_{j k a}=0, \\
& X R^{i}{ }_{j k l ; m} \equiv \xi^{a} R^{i}{ }_{j k l ; m ; a}-\xi^{i}{ }_{a} R^{a}{ }_{j k l ; m}+\xi^{a}{ }_{; j} R^{i}{ }_{a k l ; m}+\xi^{a}{ }_{i k} R^{i}{ }_{j a l ; m} \\
& +\xi^{a}{ }_{i l} R^{i}{ }_{j k a ; m}+\xi^{a}{ }_{i m} R^{i}{ }_{j k l ; a}=0,
\end{aligned}
$$

Thus, in order that system (3.7) of partial differential equations be integrable, it is necessary and sufficient that there exist a positive integer $N$ such that equations $(3.8)_{1},(3.8)_{2}, \cdots,(3.8)_{N+1}$ are automatically satisfied by $\xi^{i}$ and $\xi^{i} ; j$ satisfying equations $(3.8)_{1},(3.8)_{2}, \cdots,(3.8)_{N}$.

In this case, if $N$ equations $(3.8)_{1},(3.8)_{2}, \cdots,(3.8)_{N}$ give just $r$ linearly independent equations with respect to $\xi^{i}$ and $\xi^{i} ; j$, then the space admits a group of affine collineations with dimension $n^{2}+n-r$.

Conversely, if the space admits a group of affine collineations with dimension $n^{2}+n-s$, then there exist $s$ linearly independent relations between $\xi^{i}$ and $\xi^{i}{ }_{i j}$ and the integrability conditions $(3.8)_{1}, 3.8_{2}, \ldots$ should be automatically satisfied by $\xi^{i}$ and $\xi^{i} ;$ satisfying these $s$ relations $[9 ; 15]$.

Now, when the space admits a group $G$ of affine collineations with dimension $r$, if we take a point $P$ in the space and consider all the transformations of the group which fix this point $P$, then such transformations form a sub- 
group $G_{P}$, called the isotropic subgroup at $P$. This subgroup $G_{P}$ consists of transformations

$$
T: \bar{x}^{i}=f^{i}(x ; \alpha)
$$

such that

$$
x_{0}^{i}=f^{i}\left(x_{0} ; \alpha\right)
$$

where $x_{0}^{i}$ are the coordinates of the points $P$, and $\alpha$ denotes the parameters.

To each transformation $T_{\alpha}$ in $G_{P}$, there corresponds a linear transformation

$$
\tau\left(T_{\alpha}\right): d \bar{x}^{i}=\frac{\partial f^{i}\left(x_{0} ; \alpha\right)}{\partial x^{j}} d x^{j}
$$

of the tangent space $\mathcal{L}_{P}$ at the point $P$. It can be easily proved that this linear representation $\tau$ of $G_{P}$ is an isomorphism in the sense of topological groups [16].

Now consider the matrix $\left(\xi_{a}^{q}\right)(a=1,2, \cdots, r)$ of the components of a basis of the infinitesimal group of $G$, and denote by $q$ the generic rank of this matrix. A point is called an ordinary point if, at this point, the matrix assumes the maximum rank $q$, and is called a singular point if otherwise.

Let $\mathfrak{A}_{n}$ be an $n$-dimensional space with a symmetric affine connection admitting a group $G$ of affine collineations of dimension greater than or equal to $n^{2}-n+5$. We confine ourselves to an open domain containing only ordinary points. Let $G_{P}$ denote the isotropic subgroup at $P$. Then evidently $\operatorname{dim} G_{P}$ $=\operatorname{dim} \tau\left(G_{P}\right) \geqq n^{2}-2 n+5$. Thus, by corollary to Theorem 1 , the identity component $A_{P}$ of $\tau\left(G_{P}\right)$ should be conjugate to one of the groups $H_{n}^{+}, P$, $K \times M, K \times M^{\prime}, K \times L, K \times L^{\prime}, I(b) \times L, I(b) \times L^{\prime}, L, L^{\prime}$.

4. The case by which $A_{P}$ is conjugate to $H_{n}^{+}$or $P_{n}$. In these two cases, the group $G$ is transitive. Because if $G$ is not transitive, there would be an invariant subvariety passing through $P$, and consequently, $A_{P}$ would leave invariant a proper linear subspace of the tangent space $\mathcal{L}_{P}$ at the point $P$ which is impossible.

1. CASE $A_{P}=H_{n}^{+}$. In this case, $G$ is of dimension $n^{2}+n$. Thus the integrability conditions $(3.8)_{1}$ should be satisfied identically by any $\xi^{i}$ and $\xi^{i} ; j$. Thus by writing $(3.8)_{1}$ in the form

$$
\xi^{a} R^{i}{ }_{j k l i a}-\xi_{; a}^{b}\left(\delta_{b}^{i} R^{a}{ }_{j k l}-\delta_{j}^{a} R^{i}{ }_{b k l}-\delta_{k}^{a} R^{i}{ }_{j b l}-\delta_{l}^{a} R^{i}{ }_{j k b}\right)=0\left({ }^{4}\right),
$$

we obtain

$$
R^{i}{ }_{j k l ; a}=0
$$

and

$$
\delta_{b}^{i} R^{a}{ }_{j k l}-\delta_{j}^{a} R^{i}{ }_{b k l}-\delta_{k}^{a} R^{i}{ }_{j b l}-\delta_{l}^{a} R^{i}{ }_{j k b}=0 .
$$


From (4.3), we get, by contraction with respect to $a$ and $b$,

$$
R^{i}{ }_{j k l}=0 .
$$

It is evident that if $R^{i}{ }_{j k l}=0$, then conditions $(3.8)_{1},(3.8)_{2}, \cdots$ are automatically satisfied. Thus, in order that $A_{P}=H_{n}^{+}$, it is necessary and sufficient that the space is flat.

2. CASE $A_{P}=P_{n}$. In this case, the group $A_{P}$ being of dimension $n^{2}-1$ and the group $G$ being transitive, we know that $\operatorname{dim} G=n^{2}+n-1$. Since $A_{P}=P_{n}$, $\xi^{i} ; j$ should satisfy

$$
\xi^{a} ; a=0
$$

and the integrability conditions (3.8) should be satisfied identically by any $\xi^{i}$ and $\xi^{i} ; j$ satisfying (4.5).

Thus comparing (3.8) ${ }_{1}$ and (4.5), we see that there should exist functions $F^{i}{ }_{j k l}$ such that

$$
\xi^{a} R^{i}{ }_{j k l ; a}-\xi^{i}{ }_{; a} R^{a}{ }_{j k l}+\xi^{a}{ }_{j j} R^{i}{ }_{a k l}+\xi_{; k}^{a} R^{i}{ }_{j a l}+\xi^{a}{ }_{i l} R^{i}{ }_{j k a}=-\xi^{a}{ }_{; a} F^{i}{ }_{j k l}
$$

become identities in $\xi^{i}$ and $\xi^{i} ;$. Thus we must have

$$
R^{i}{ }_{j k l ; a}=0
$$

and

$$
\delta_{b}^{i} R^{a}{ }_{j k l}-\delta_{j}^{a} R^{i}{ }_{b k l}-\delta_{k}^{a} R^{i}{ }_{j b l}-\delta_{l}^{a} R^{i}{ }_{j k b}=\delta_{b}^{a} F^{i}{ }_{j k l} .
$$

By contraction with respect to $a$ and $b$, we find from (4.7)

$$
F^{\boldsymbol{i}_{j k l}}=-(2 / n) \boldsymbol{R}^{\boldsymbol{i}}{ }_{j k l},
$$

and by contraction with respect to $i$ and $b$, we get

$$
n R^{a}{ }_{j k l}-\delta_{l}^{a} R_{j k}+\delta_{k}^{a} R_{j l}+\delta_{j}^{a}\left(R_{k l}-R_{l k}\right)=-(2 / n) R^{a}{ }_{j k l},
$$

where $R_{j k}=R^{a}{ }_{j k a}$. Contracting again with respect to $a$ and $l$, we find $R_{j k}=0$ for $n>2$. Thus we have, from (4.8), $R^{i}{ }_{j k l}=0$.

5. The case in which $A_{P}$ is conjugate to $K \times M, K \times M^{\prime}, K \times L$ or $K \times L^{\prime}$. In these cases, the group $G$ is transitive. We shall prove this by method of contradiction.

We first suppose that $A_{P}=K \times M$ or $K \times L$ and that the group $G$ is intransitive. Then the invariant subvariety passing through $P$ should be onedimensional, because the linear manifold tangent to this subvariety at $P$ is left invariant by $K \times M$ or $K \times L$ which fixes one and only one direction. Thus the rank of the matrix $\left(\xi_{a}^{i}\right)$ is equal to 1 at $P$ and, consequently, is equal to 1 at every point of the domain under consideration. It follows that through every point of his domain there passes one and only one invariant curve.

Now take an invariant curve passing through a point $Q$ which is not on the invariant curve passing through $P$ and which is in the domain under 
consideration, and consider all the paths joining $P$ to the points on the invariant curve passing through $Q$. These paths constitute a two-dimensional surface. This surface is left invariant by the isotropic subgroup $G_{P}$. Consequently, the corresponding linear group $A_{P}$ must fix the two-dimensional plane tangent to this surface at $P$ which contradicts our assumption.

We next suppose that $A_{P}=K \times M^{\prime}$ or $K \times L^{\prime}$ and that the group $G$ is intransitive. The invariant subvariety passing through $P$ should be $(n-1)$ dimensional, because the linear manifold tangent to this subvariety at $P$ is left invariant by $K \times M^{\prime}$ or $K \times L^{\prime}$ which fixes one and only one hyperplane. Thus the rank of the matrix $\left(\xi_{a}^{\ell}\right)$ is equal to $n-1$ at $P$ and, consequently, is equal to $n-1$ at every point of the domain under consideration. It follows that through every point of the domain there passes one and only one invariant hypersurface.

Now, consider a path through $P$ which intersects these invariant hypersurfaces; then the points of intersections can be transformed by $K$ into one another (except the point $P$, of course), which is a contradiction.

Thus, in these cases, the group $G$ is transitive, and consequently two isotropic groups at any two ordinary points in the domain under consideration are conjugate to each other.

The groups $K \times M, K \times M^{\prime}, K \times L, K \times L^{\prime}$ being respectively with dimension $n^{2}-n+1, n^{2}-n+1, n^{2}-n, n^{2}-n$, and the group $G$ being transitive, the group $G$ is respectively with dimension $n^{2}+1, n^{2}+1, n^{2}, n^{2}$.

Now, at the point $P$ of the domain, we choose the normal coordinates $x^{i}$ whose origin is $P$, then the space admits a one-parameter group of affine collineations

$$
\bar{x}^{i}=e^{t} x^{i} .
$$

In this coordinate system, the vector $\xi^{i}$ defining the infinitesimal transformation of this one-parameter group is given by

$$
\xi^{i}=x^{i} .
$$

Thus, the integrability condition $(3.8)_{1}$ becomes

$$
x^{a} \frac{\partial R^{i}{ }_{j k l}}{\partial x^{a}}+2 R^{i}{ }_{j k l}=0
$$

which shows that $R^{i}{ }_{j k l}$ are homogeneous functions of degree -2 of $x^{i}$.

But we know that the components $R^{i}{ }_{j k l}$ of the curvature tensor are well defined at the origin of the normal coordinates system. Thus the components $R^{i}{ }_{j k l}$ must vanish at $P$ and consequently at any point of the domain. Thus, in these cases, the space is affinely flat.

6. The case in which $A_{P}$ is conjugate to $I(b) \times L$ or $L$. In these cases, the group $G$ is transitive. This can be proved by the same argument as that used at the beginning of $\S 5$. 
The group $G$ being transitive, the isotropic groups at any two points of the domain under consideration are conjugate to each other. On the other hand, the isotropic group $G_{Q}$ at an ordinary point $Q$ fixes one and only one direction which we denote by $u_{Q}$. Thus, at every point $Q$ of the domain under consideration, there is associated a direction $u_{Q}$.

Consider a path which passes through a point $Q$ and tangent to $u_{Q}$; then the isotropic group $G_{Q}$, being an affine collineation, fixes this path. We take a point $R$ different from $Q$ on this path and consider the transformations of $G_{Q}$ which fix this point $R$. These transformations form the group $L$.

Now, we consider an affine frame at $Q$ whose first axis is in the direction $u_{Q}$ and transport it parallelly along the path to the point $R$. Then we have at $R$ an affine frame whose first axis is tangent to the path. The parallelism of vectors along a curve being preserved by an affine collineation, the transformation of $G_{Q}$ fixing the point $R$ gives the same effect on the affine frame at $R$ as on that at $Q$. This shows that the subgroup of $G_{Q}$ fixing $R$ coincides with the subgroup of $G_{R}$ fixing $Q$. The subgroup of $G_{R}$ fixing $Q$ fixes the tangent to the path and $u_{R}$, and consequently the tangent must coincide with $u_{R}$, which shows that the path is the trajectory of the field of directions $u$.

Now, the isotropic groups $I(b) \times L$ and $L$ being respectively with dimension $n^{2}-n$ and $n^{2}-n-1$, and the group $G$ being transitive, the group $G$ is respectively with dimension $n^{2}$ and $n^{2}-1$.

Now, the group $G$ of affine collineations being transitive, we denote by $T$ a transformation of $G$ which carries a point $Q$ into a point $R$. Then, by the same method as in [16], we can prove that

$$
T u_{Q}=u_{R}
$$

and that $u_{Q}$ is a parallel vector field.

If we denote this vector field by $u^{i}(x)$, then we have

$$
\begin{aligned}
& X u^{i}=\alpha u^{i}, \\
& u^{i} ; k=u^{i} \lambda_{k},
\end{aligned}
$$

where $\alpha$ is a certain scalar and $\lambda_{k}$ a certain covariant vector field. From (6.2), we find

$$
u^{i} R^{i}{ }_{j k l}=u^{i} \lambda_{k l}
$$

where

$$
\lambda_{k l}=\lambda_{k ; l}-\lambda_{l ; k} .
$$

We first suppose that $A_{P}=I(b) \times L$. Then equations $(3.8)_{1}$ should be satisfied by any $\xi^{i}$ and $\xi^{i}{ }_{i j}$ satisfying

$$
(1+n b) X u^{i}=(1+b) \xi_{; a}^{a} u^{i} .
$$

We see that conditions 


$$
X u^{i} \equiv \xi^{a} u_{; a}^{i}-\xi_{; a}^{i} u^{a}=0
$$

and

$$
\xi_{; a}^{a}=0
$$

put together are stronger than (6.5). Hence any $\xi^{i}$ and $\xi^{i} ; j$ satisfying (6.6) and (6.7) must satisfy (6.5) and hence satisfy (3.8) $)_{1}$.

The group being that of affine collineations, the covariant differentiation and the Lie derivation are commutative and consequently, from (6.2) and (6.6), we find $X \lambda_{k}=0$. But the group $G_{P}$ does not fix a hyperplane and consequently we should have $\lambda_{k}=0$. Consequently we have

$$
u^{i} ; k=0 \text { and } u^{i} R^{i}{ }_{j k l}=0 .
$$

Thus the integrability conditions $(3.8)_{1}$ should be satisfied by any $\xi^{i}$ and $\xi^{i} ; j$ satisfying

$$
\xi_{; a}^{i} u^{a}=0 \text { and } \xi_{; a}^{a}=0
$$

and consequently there must exist functions $F^{i}{ }_{j k l}$ and $G^{i}{ }_{j k l b}$ such that

$$
R^{i}{ }_{j k l ; a}=0
$$

and

$$
\delta_{b}^{i} R^{a}{ }_{j k l}-\delta_{j}^{a} R_{b k l}^{i}-\delta_{k}^{a} R^{i}{ }_{j b l}-\delta_{l}^{a} R^{i}{ }_{j k b}=\delta_{b}^{a} F^{i}{ }_{j k l}+u^{a} G^{\boldsymbol{i}}{ }_{j k l b} .
$$

From (6.11), after some calculation, we can deduce $R^{i}{ }_{j k l}=0$.

The case $A_{P}=L$ is characterized by (6.6) and (6.7) and consequently the above discussion shows that when $A_{P}=L$ the space is also affinely flat.

7. The case in which $A_{P}$ is conjugate to $I(b) \times L^{\prime}$ or $L^{\prime}$ and $G$ is transitive. The group $G$ being transitive, two isotropic groups at any two ordinary points in the domain under consideration are conjugate to one another.

On the other hand, the isotropic group $G_{Q}$ at an ordinary point $Q$ fixes one and only one hyperplane which we denote by $v_{Q}$. Thus with every point $Q$ of the domain under consideration, there is associated a hyperplane $v_{\boldsymbol{Q}}$.

The isotropic groups $I(b) \times L^{\prime}$ and $L^{\prime}$ being respectively with dimension $n^{2}-n$ and $n^{2}-n-1$ and the group $G$ being transitive, the group $G$ is respectively with dimension $n^{2}$ and $n^{2}-1$.

By exactly the same method as in [16], we can prove that

$$
T v_{Q}=v_{R},
$$

where $T$ is an arbitrary transformation carrying a point $Q$ into a point $R$.

Furthermore, if we represent this hyperplane by a covariant vector $v_{j}(x)$, then we can prove that

$$
\begin{gathered}
X v_{j}=\beta v_{j}, \\
v_{j ; k}=\alpha v_{j} v_{k},
\end{gathered}
$$


where $\alpha$ is a certain scalar. From (7.2), we find

$$
-v_{i} R_{j k l}=v_{j} \alpha_{k l},
$$

where

$$
\alpha_{k l}=v_{k} \alpha_{; l}-v_{l} \alpha_{; k} .
$$

We first suppose that $A_{P}=I(b) \times L^{\prime}$. Then equations (3.8) $)_{1}$ should be satisfied by any $\xi^{\boldsymbol{i}}$ and $\xi^{\boldsymbol{i}} ;$ i satisfying

$$
(1+n b) X v_{j}=(1+b) \xi_{; a}^{a} v_{j} .
$$

We see that conditions

$$
\begin{aligned}
& X v_{j}=\xi^{a} \alpha v_{j} v_{a}-\xi^{a}{ }_{; j} v_{a}=0, \\
& \xi^{a}{ }_{; a}=0
\end{aligned}
$$

put together are stronger than (7.5). Hence any $\xi^{i}$ and $\xi^{i} ; j$ satisfying (7.6) and (7.7) must satisfy (7.5) and hence satisfy (3.8) ${ }_{1}$.

The group being that of affine collineations, the covariant differentiation and the Lie derivation are commutative, and consequently, from (7.2) and (7.6) we find $X \alpha=0$, which shows, the group $G$ being transitive, that $\alpha$ is a constant.

Thus the integrability conditions (3.8) 1 should be satisfied by any $\xi^{i}$ and $\xi^{i} ; j$ satisfying (7.6) and (7.7) and consequently there must exist functions $F^{i}{ }_{j k l}$ and $G^{i}{ }_{j k l}{ }^{a}$ such that

$$
R^{i}{ }_{j k l ; a}=-\alpha G^{i}{ }_{j k l}{ }^{c} v_{c} v_{a}
$$

and

$$
\delta_{b}^{i} R^{a}{ }_{j k l}-\delta_{j}^{a} R^{i}{ }_{b k l}-\delta_{k}^{a} R^{i_{j b l}}-\delta_{l}^{a} R^{i}{ }_{j k b}=\delta_{b}^{a} F^{i}{ }_{j k l}+v_{b} G^{i}{ }_{j k l}^{a} .
$$

From (7.9), after some calculation, we can conclude that

$$
R^{\boldsymbol{i}_{j k l}}=k v_{j}\left(v_{k} \delta^{\boldsymbol{i}_{l}}-v_{l} \delta^{\left.\boldsymbol{i}{ }_{k}\right)},\right.
$$

where $k$ is a constant. Thus equations (3.8), become

$$
X R^{i}{ }_{j k l}=\beta R^{i}{ }_{j k l},
$$

where $\beta$ is given by $X v_{j}=\beta v_{j}$.

When $1+b \neq 0$ there exists $X$ such that $\beta \neq 0$ and thus we have $R^{i}{ }_{j k l}=0$. When $1+b=0$ then $X v_{j}=0$ and thus $(3.8)_{1}$ is really satisfied by all the infinitesimal transformations $X$ of the group $G$.

8. The case in which $A_{P}$ is conjugate to $I(b) \times L^{\prime}$ or $L^{\prime}$ and $G$ is intransitive. Let us consider the invariant variety through $P$. All the points on this invariant variety being equivalent under the group $G$, isotropic groups at points of this invariant variety are conjugate to each other. Thus the invariant variety should be $(n-1)$-dimensional, because the hyperplane tan- 
gent to this invariant variety at a point should be left invariant by the isotropic group $I(b) \times L^{\prime}$ or $L^{\prime}$ at this point which fixes one and only one hyperplane.

Take a point $Q$ not on this invariant variety. If the isotropic group at $Q$ is one of the groups hitherto examined except $I(b) \times L^{\prime}$ and $L^{\prime}$, then the group $G$ should be transitive. Thus the isotropic group at $Q$ should be also $I(b) \times L^{\prime}$ or $L^{\prime}$.

Consequently, passing through every ordinary point on the domain under consideration, there exists an $(n-1)$-dimensional invariant variety whose tangent hyperplane is fixed by the isotropic group at the point of contact. We denote 'this hyperplane at $Q$ by $v_{Q}$.

The isotropic group $I(b) \times L^{\prime}$ and $L^{\prime}$ being respectively with dimension $n^{2}-n$ and $n^{2}-n-1$, and the invariant varieties being $(n-1)$-dimensional, the group $G$ is respectively with dimension $n^{2}-1$ and $n^{2}-2$.

Thus if we denote by

$$
f(x)=\text { constant }
$$

the family of invariant varieties and put

$$
\lambda v_{j}=\partial f / \partial x^{j},
$$

then, using the so-called adopted frames, we can prove that

$$
\left(\lambda v_{j}\right)_{; k}=\left(\lambda v_{j}\right) p_{k}+\left(\lambda v_{k}\right) p_{j}
$$

$p_{k}$ being a certain covariant vector.

On the other hand, we know that

$$
X f=0, \quad X\left(\lambda v_{j}\right)=0, \quad X\left(\lambda v_{j}\right)_{; k}=0
$$

and consequently, from (8.3), we find

$$
X p_{k}=0 .
$$

But the hyperplane represented by $v_{j}$ is the only one hyperplane fixed by the isotropic group and consequently, we should have

$$
p_{k}=(1 / 2) \alpha v_{k}
$$

where $\alpha$ is a certain function of $f$.

Thus substituting this into (8.3), we get

$$
\left(\lambda v_{j}\right)_{i k}=\alpha\left(\lambda v_{j}\right)\left(\lambda v_{i k}\right)
$$

from which

$$
v_{i} R^{i}{ }_{j k l}=0 .
$$

We first suppose that $A_{P}=I(b) \times L^{\prime}$. Then equations (3.8) ${ }_{1}$ should be satisfied by any $\xi^{i}$ and $\xi^{i} ;$; satisfying 


$$
(1+n b) X v_{j}=(1+b) \xi^{a}{ }_{i a} v_{j}
$$

We see that conditions

$$
\begin{aligned}
X f & \equiv \lambda v_{a} \xi^{a}=0, \\
X v_{j} & \equiv \xi^{a} v_{j ; a}+\xi_{; j}^{a} v_{a}=0, \\
\xi^{a} ; a & =0
\end{aligned}
$$

put together are stronger than (8.6). Hence any $\xi^{i}$ and $\xi^{i}{ }_{i j}$ satisfying (8.7), (8.8), and (8.9) must satisfy (8.6) and hence satisfy $(3.8)_{1}$.

Equations $X\left(\lambda v_{j}\right)=0$ and $X v_{j}=0$ show that $X \lambda=0$ and consequently that $\lambda$ is a function of $f$. Thus, from (8.2), we can see that we can suppose $\lambda=1$. Thus equation (8.8) can be written as

$$
X v_{j} \equiv \xi_{; j}^{a} v_{a}=0
$$

by virtue of (8.4) and (8.7).

Thus the integrability conditions $(3.8)_{1}$ should be satisfied by any $\xi^{i}$ and $\xi^{i} ; j$ satisfying (8.7), (8.9), and (8.10) and consequently there must exist functions $E^{i_{j k l},}, F^{i}{ }_{j k l}$ and $G^{\boldsymbol{i}_{j k}} t^{a}$ such that

$$
\begin{gathered}
R^{i_{j k l i a}}=E^{i_{j k l} \nu_{a}} \\
\delta_{b}^{i} R^{a}{ }_{j k l}-\delta_{j}^{a} R_{b k l}^{i}-\delta_{k}^{a} R^{i}{ }_{j b l}-\delta_{l}^{a} R^{i}{ }_{j k b}=\delta_{b}^{a} F^{i}{ }_{j k l}+G^{i}{ }_{j k l}{ }^{a} v_{b} .
\end{gathered}
$$

From (8.12) we can conclude that the curvature tensor $R^{i}{ }_{j k l}$ should be of the form

$$
R^{i}{ }_{j k l}=k v_{j}\left(v_{k} \delta_{l}^{i}-v_{l} \delta_{k}^{i}\right) \text {. }
$$

But since we have $X R^{i}{ }_{j k l}=0, X v_{j}=0$, we find from this $X k=0$, which shows that $k$ is a certain function of $f$.

Thus equations $(3.8)_{1}$ become

$$
X R^{i_{j k l}}=\beta R^{i_{j k l}},
$$

where $\beta$ is given by $X v_{j}=\beta v_{j}$. When $1+b \neq 0$, there exists $X$ such that $\beta \neq 0$ and thus $R^{i}{ }_{j k l}=0$. When $1+b=0$, then $X v_{j}=0$ and thus $(3.8)_{1}$ is really satisfied by all the infinitesimal transformations $X$ of the group $G$.

The case $A_{P}=L^{\prime}$ is characterized by (8.7), (8.8), and (8.9) also and consequently the above discussion shows that when $A_{P}=L^{\prime}$, the space has also the curvature tensor of the form (8.13).

9. Theorems. Gathering all the results in $\$ \$ 3-8$, we have

THEOREM 2. If an $n$-dimensional space with a symmetric affine connection admits a group of affine collineations with dimension greater than $n^{2}-n+5$, then the isotropic group $G_{P}$ at a point $P$, the dimension of $G_{P}$, the groups of affine collineations $G$, the dimension of $G$, and the structure of the space should be one of those on the opposite page: 
isotropic dimension group of dimension group $G_{P}$ of $G_{P}$ lineations $G$

$H_{n} \quad n^{2}$

$H_{n}^{+} \quad n^{2}$

$P_{n} \quad n^{2}-1$

$K \times M \quad n^{2}-n+1$

$K \times M^{\prime} \quad n^{2}-n+1$

$K \times L \quad n^{2}-n$

$K \times L^{\prime} \quad n^{2}-n$

$I(b) \times L \quad n^{2}-n$

$L \quad n^{2}-n-1$

$I(b) \times L^{\prime} \quad n^{2}-n$

tran

$n^{2}+n$

\begin{tabular}{|c|}
\hline 21 \\
\hline$n^{2}+n-1$ \\
\hline
\end{tabular}

$n^{2}+1$

$n^{2}+1$

$n^{2}$

“

“

“

“

“

“

6

$n^{2}-1$

$n^{2}$

affinely flat

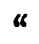

structure of the space

(i) $1+b \neq 0$,

$R^{i}{ }_{j k l}=0$.

(ii) $1+b=0$,

$v_{j ; k}=\alpha v_{j} v_{k}$,

$R_{j k l}^{i_{j k}}=k v_{j}\left(v_{k} \delta_{l}^{i}-v_{l} \delta_{k}^{i}\right)$,

$\alpha, k$ : constants.

intransitive $\quad n^{2}-1$

(i) $1+b \neq 0$,

$R^{\boldsymbol{i}_{j k l}}=\mathbf{0}$.

(ii) $1+b=0$,

$v_{j ; k}=\alpha v_{j} v_{k}$,

$\left(v_{j}=\frac{\partial f}{\partial x^{j}}\right)$,

$R^{i}{ }_{j k l}=k v_{j}\left(v_{k} \delta_{l}^{i}-v_{l} \delta_{k}^{i}\right)$,

$\alpha, k$ : functions of $f$.

$L^{\prime} \quad n^{2}-n-1 \quad$ transitive $\quad n^{2}-1$

$v_{j ; k}=\alpha v_{j} v_{k}$,

$R_{j k l}^{i}=k v_{j}\left(v_{k} \delta_{l}^{i}-v_{l} \delta_{k}^{i}\right)$,

$\alpha, k$ : constants.

intransitive $n^{2}-2 \quad v_{j ; k}=\alpha v_{j} v_{k}$,

$\left(v_{j}=\frac{\partial f}{\partial x^{j}}\right)$,

$R_{j k l}^{i}=k v_{j}\left(v_{k} \delta_{l}^{i}-v_{l} \delta_{k}^{i}\right)$,

$\alpha, k$ : functions of $f$. 


\section{Determination of $\mathfrak{A}_{n}$ when $G$ is transitive.}

THEOREM 3. Let $\mathfrak{A}_{n}$ be a simply-connected, n-dimensional manifold with a symmetric affine connection. Suppose that $\mathfrak{A}_{n}$ admits a group $G$ of affine collineations with $\operatorname{dim} G \geqq n^{2}-n+5$. Let $\mathfrak{B}_{n}$ denote the entire coordinate space with coordinates $x^{1}, x^{2}, \cdots, x^{n}$, and let $i, j, k, \cdots$ be indices running from 1 to $n$ while $\alpha, \beta$ indices running from 2 to $n$. Then $\mathfrak{A}_{n}$ is equivalent to one of those on the following page. Moreover, the affinely connected spaces listed above are nonequivalent.

Before proving the theorem, we shall firstly give three remarks and establish a lemma.

REMARK 1. Let $G^{*}$ be the group of all affine collineations of $\mathfrak{A}_{n}$. Then with respect to a suitable topology, $G^{*}$ forms a Lie group $\left({ }^{5}\right)$. Thus the closure of any subgroup of $G^{*}$ is a Lie group. For this reason, we can always assume the group $G$ in Theorem 3 to be a connected Lie group, for otherwise, we can take the identity component of the closure of $G$ instead of $G$. This does not effect the transitivity and the dimension restriction.

REMARK 2. Let $G_{p}$ be the isotropic subgroup of $G$ at a point $p$, and $G, G_{p}$ be the Lie algebras of $G, G_{p}$ respectively. Since $\mathfrak{A}_{n}$ is simply-connected, the space $\mathfrak{A}_{n}$ as well as the action of $G$ on $\mathfrak{A}_{n}$ is uniquely determined by the pair $\left(G, G_{p}\right)$.

REMARK 3. Let $E$ be the quotient $G / G_{p}$ in the sense of linear space, and $\phi: G \rightarrow E$ the natural linear mapping. Denoting respectively by "Ad" and "ad" the linear adjoint representations of $G$ and $G$ over $G$, we have

$$
\operatorname{Ad}\left(G_{p}\right)\left(G_{p}\right) \subset G_{p}, \text { ad }\left(G_{p}\right)\left(G_{p}\right) \subset G_{p} .
$$

Thus Ad and ad induce linear representations $\Psi$ and $\psi$ of $G_{p}$ and $G_{p}$ over $E$ respectively. In fact,

$$
\begin{aligned}
& \Psi(u)(b)=\phi \operatorname{Ad}(u) \phi^{-1}(b), \quad \psi(x)(b)=\phi \operatorname{ad}(x) \phi^{-1}(b), \\
& u \in G_{p}, \quad x \in G_{p}, \quad b \in E .
\end{aligned}
$$

On the other hand, there is a natural 1-1 linear correspondence between $E$ and the tangent space $\mathcal{L}_{p}$ of $\mathfrak{A}_{n}$ at $p$. Up to this correspondence,

$$
\Psi\left(G_{p}\right)=\tau\left(G_{p}\right) .
$$

\section{A lemma.}

Lemma. Let $P_{t}$ denote the Lie algebra of the real special linear group of degree $t$, and $\mathbf{S}$ a semi-simple Lie algebra containing $\boldsymbol{P}_{r}$ as a subalgebra. If $\operatorname{dim} \mathbf{S}$ $\leqq r^{2}+2 r+2, r \geqq 4$, then the least ideal $S_{m}$ of $S$ such that $P_{r} \subset S_{m}$ is either $P_{r}$ itself or $a P_{r+1}$.

(๖) Cf. S. Kobayashi: Groupe de transformations qui laissent invariante une connexion infinitesimale, C. R. Acad. Sci. Paris vol. 238 (1954) pp. 644-645. 


\begin{tabular}{|c|c|c|c|}
\hline Types & 1 & 2 & 3 \\
\hline Spaces & $\mathfrak{B}_{n}$ & $\mathfrak{V}_{n}$-origin & $\mathfrak{B}_{n}$ \\
\hline Connections & $\Gamma_{j k}^{i}(x)=0$ & $\Gamma_{j k}^{i}(x)=0$ & $\begin{array}{c}\Gamma_{11}^{1}(x)=2, \Gamma_{1 \alpha}^{\beta}(x)=\delta_{\alpha}^{\beta} \\
\text { others }=0\end{array}$ \\
\hline $\begin{array}{l}\text { Maximal group } \\
G^{*} \text { of colline- } \\
\text { ations }\end{array}$ & $\begin{array}{c}y^{i}=C_{0}^{i}+C_{k}^{i x^{k}}, \\
\left|C_{k}^{i}\right| \neq 0\end{array}$ & $\begin{array}{l}y^{i}=C_{k}^{i k} x^{k} \\
\left|C_{k}^{i}\right| \neq 0\end{array}$ & $\mid \begin{array}{l}y^{1}=x^{1}+C_{0}^{1}, \quad\left|C_{\alpha}^{\beta}\right| \neq 0, \\
y^{\alpha}=C_{0}^{\alpha}+C_{1}^{\alpha} x^{1}+C_{\beta}^{\alpha} x^{\beta}\end{array}$ \\
\hline $\operatorname{dim} G^{*}$ & $n^{2}+n$ & $n^{2}$ & $n^{2}$ \\
\hline$R_{j k l}^{i_{j k l}}$ & 0 & 0 & $\delta_{j}^{1}\left(\delta_{l}^{i} \delta_{k}^{1}-\delta_{k}^{i} \delta_{l}^{1}\right)$ \\
\hline Completeness & complete & not & not \\
\hline
\end{tabular}

\begin{tabular}{|c|c|c|}
\hline Types & 4 & 5 \\
\hline Spaces & $\mathfrak{B}_{n}$ & $\mathfrak{B}_{n}$ \\
\hline Connections & $\begin{array}{l}\Gamma_{11}^{1}=2 k, \Gamma_{1 \beta}^{\alpha}=k \delta_{\beta}^{\alpha}, \Gamma_{11}^{\alpha}=-x^{\alpha} \\
\text { others }=0, \quad k=\text { constant } \geqq 0\end{array}$ & $\begin{array}{l}\Gamma_{11}^{1}=2 k, \Gamma_{1 \beta}^{\alpha}=k \delta_{\beta}^{\alpha}, \Gamma_{11}^{\alpha}=x^{\alpha} \\
\text { others }=0, k=\text { constant } \geqq 0\end{array}$ \\
\hline $\begin{array}{l}\text { Maximal group } \\
G^{*} \text { of colline- } \\
\text { ations }\end{array}$ & $\begin{aligned} y^{1}= & x^{1}+C_{0}^{1},\left|C_{\beta}^{\alpha}\right| \neq 0 \\
y^{\alpha}= & C_{0}^{\alpha} \cosh x^{1}+C_{1}^{\alpha} \sinh x^{1} \\
& +C_{\beta}^{\alpha} x^{\beta}\end{aligned}$ & $\begin{aligned} y^{1}= & x^{1}+C_{0}^{1},\left|C_{\beta}^{\alpha}\right| \neq 0 \\
y^{\alpha}= & C_{0}^{\alpha} \cos x^{1}+C_{1}^{\alpha} \sin x^{1} \\
& +C_{\beta}^{\alpha} x^{\beta}\end{aligned}$ \\
\hline $\operatorname{dim} G^{*}$ & $n^{2}$ & $n^{2}$ \\
\hline$R_{j k l}^{i}$ & $\left(k^{2}-1\right) \delta_{j}^{1}\left(\delta_{l}^{i} \delta_{k}^{1}-\delta_{k}^{i} \delta_{l}^{1}\right)$ & $\left(k^{2}+1\right) \delta_{j}^{1}\left(\delta_{l}^{i} \delta_{k}^{1}-\delta_{k}^{i} \delta_{l}^{1}\right)$ \\
\hline Completeness & complete when $k=0$ & complete when $k=0$ \\
\hline
\end{tabular}


Proof. Evidently $S_{m}$ is semi-simple and $\operatorname{dim} S_{m} \leqq r^{2}+2 r+2$. Let $S_{m}^{*}$ be the complex form of $S_{m}$, and $P_{r}^{*}$ the subalgebra of $S_{m}^{*}$ which corresponds to $P_{r}$. We know that (1) $S_{m}^{*}$ is semi-simple, (2) $P_{r}^{*}$ is the complex simple Lie algebra of class A and rank $r-1$, (3) no proper ideal of $S_{m}^{*}$ can contain $P_{r}^{*}$. Suppose that $S_{m}^{*}$ is not simple. Then we can write it as a direct sum

$$
S_{m}^{*}=S_{1}^{*} \oplus S_{2}^{*}
$$

of two semi-simple nontrivial ideals. The intersection $S_{1}^{*} \cap P_{r}^{*}$ must be zero, for otherwise, we obtain from the simplicity of $P_{r}^{*}$ that $P_{r}^{*} \subset S_{1}^{*}$ which is impossible. Similarly, $S_{2}^{*} \cap P_{r}^{*}=0$. It follows then that $\pi_{1}\left(P_{r}^{*}\right) \approx P_{r}^{*}, \pi_{2}\left(P_{r}^{*}\right)$ $\approx P_{r}^{*}$, where $\pi_{1}: S_{m}^{*} \rightarrow S_{1}^{*}, \pi_{2}: S_{m}^{*} \rightarrow S_{2}^{*}$ are the projections. Thus

$$
\operatorname{dim} S_{1}^{*} \geqq \operatorname{dim} P_{r}^{*}=r^{2}-1, \quad \operatorname{dim} S_{2}^{*} \geqq \operatorname{dim} P_{r}^{*}=r^{2}-1,
$$

whence

$$
2 r^{2}-2 \leqq \operatorname{dim} S_{1}^{*}+\operatorname{dim} S_{2}^{*}=\operatorname{dim} S_{m}^{*} \leqq r^{2}+2 r+2 .
$$

But this contradicts our hypothesis that $r \geqq 4$. Hence $S_{m}^{*}$ must be simple.

Since $P_{r}^{*} \subset S_{m}^{*}$, we know that

$$
r^{2}-1 \leqq \operatorname{dim} S_{m}^{*} \leqq r^{2}+2 r+2, \quad \operatorname{rank}\left(S_{m}^{*}\right) \geqq r-1, \quad r \geqq 4 .
$$

A survey of the list of complex simple Lie algebras tells us that $S_{m}^{*}$ has only three possibilities: (i) $S_{m}^{*}$ is of class A and rank $r-1$; (ii) $S_{m}^{*}$ is of class A and rank $r$; or (iii) $S_{m}^{*}$ is of class B or C and of rank 3 and $r=4$. But it is well known that the complex simple Lie algebras of class $B$ and $C$ of rank 3 cannot contain $\boldsymbol{P}_{4}^{*}$. Therefore, only cases (i) and (ii) can happen.

Now we return to the real Lie algebras $P_{r}$ and $S_{m}$. In case (i),

$$
\operatorname{dim} S_{m}=\operatorname{dim} S_{m}^{*}=r^{2}-1=\operatorname{dim} P_{r}
$$

whence $\boldsymbol{S}_{m}=\boldsymbol{P}_{r}$. In case (ii), $\boldsymbol{S}_{m}$ is one of the real forms of the complex simple Lie algebras of class A and rank $r$. These real forms have been completely determined by Cartan [2]. We can see immediately from the list that $P_{r+1}$ is the only such real form which can contain $\boldsymbol{P}_{\boldsymbol{r}}$. The lemma is thus proved.

12. Proof of Theorem 3. Firstly, we observe that the maximal group $G^{*}$ of collineations of the five types of spaces listed in Theorem 3 are not isomorphic. The $G^{*}$ corresponding to the first two types can be easily distinguished from the other. As for the $G^{*}$ corresponding to the spaces of types $3,4,5$, we can distinguish them by comparing the radicals of their Lie algebras. Thus two spaces belonging to different types in Theorem 3 are not equivalent. However, in each of the later two types, there are infinitely many spaces depending on $k$. We find, by a direct calculation,

$$
R^{i_{j k l ; m}}=-4 k \delta_{m}^{i} R^{i_{j k l}}
$$


From these equalities and the expression for $R^{i}{ }_{j k l}$, it follows that the absolute value of $k$ is a local scalar invariant. Thus two spaces of the type 4 or 5 with different $k(k \geqq 0)$ are not equivalent. The last sentence in Theorem 3 is proved.

Now we shall show that each $\mathfrak{A}_{n}$ satisfying the hypothesis of Theorem 3 is equivalent to one of the listed spaces. Let $p$ be a point of $\mathfrak{A}_{n}$, and let $G, G_{p}, G, G_{p}, E, \tau, \Psi, \psi$ have the same meaning as in $\$ 10$. Since $\mathfrak{A}_{n}$ is simplyconnected and $G$ connected, $G_{p}$ must be connected. Therefore, $\tau\left(G_{p}\right)=A_{p}$. We have determined all the possible linear groups $A_{p}$. The discussions for the various cases are similar. We select only three cases to study as patterns.

I. $\tau\left(G_{p}\right)=A_{p}=H_{n}^{+}$or $P_{n}$. In this case, $\tau\left(G_{p}\right)$ is transitive over the nonzero tangent vectors at $p$, and thus $G$ is transitive over the nonzero 1-elements of $\mathfrak{A}_{n}$. It follows that $\mathfrak{A}_{n}$ is complete in the sense of affine connection. From Theorem $2, \mathfrak{A}_{n}$ is affinely flat. We know that a complete, simply-connected, homogeneous affinely connected space is uniquely determined by its local properties $\left[9\right.$, p. 50]. Hence $\mathfrak{A}_{n}$ must be equivalent to the space $\mathfrak{B}_{n}$ with $\Gamma_{j k}^{i}(x)=0$.

II. $\tau\left(G_{p}\right)=A_{p}=L$. In this case, $\operatorname{dim} G=n^{2}-1$. The hypothesis $\operatorname{dim} G$ $\geqq n^{2}-n+5$ then implies $n \geqq 6$. Since $\tau$ is a faithful representation, $G_{p}$ has a Levi-decomposition of the form

$$
G_{p}=P_{n-1}+R_{1}, \quad R_{1}=\text { radical of } G_{p}, \quad \operatorname{dim} R_{1}=n-1
$$

where $P_{n-1}$ is the Lie algebra of the real special linear group of degree $n-1$. Choose a maximal semi-simple subalgebra $S$ of $G$ such that $P_{n-1} \subset S$. Let $S_{m}$ be the minimal ideal, of $S$, which contains $P_{n-1}$. By the Lemma in $\$ 11$, either $\boldsymbol{S}_{m}=\boldsymbol{P}_{n}$ or $\boldsymbol{S}_{m}=\boldsymbol{P}_{n-1}$. We shall discuss these two cases separately.

CASE II $1 . S_{m}=P_{n}$. Then $\operatorname{dim} S_{m}=n^{2}-1=\operatorname{dim} G$ whence $\boldsymbol{S}_{m}=\boldsymbol{P}_{n}=\boldsymbol{G}$. Passing the results in Theorem 1 to Lie algebras, we know that, up to an automorphism of $P_{n}, P_{n}$ has only two subalgebras of dimension $n^{2}-n-1$, i.e., the Lie algebras $L, L^{\prime}$ of $L$ and $L^{\prime}$ respectively. If $G_{p}=L^{\prime}$, then it is easy to see that $\psi\left(G_{p}\right)$ and hence $\Psi\left(G_{p}\right)$ does not have any invariant vector. This contradicts the fact that $\Psi\left(G_{p}\right)=\tau\left(G_{p}\right)$ has an invariant vector. Therefore, $G_{p}=L$.

The real special linear group $P_{n}$ acts transitively on the space $\mathfrak{M}_{n}=\mathfrak{B}_{n}-$ origin. Its isotropic subgroup at the point $(1,0, \cdots, 0)$ is the subgroup $L$. Since $n>2, \mathfrak{M}_{n}$ is simply connected. By Remark 2 in $\$ 10$, we can regard $\mathfrak{A}_{n}$ to be $\mathfrak{M}_{n}$ and the group $G$ to be $P_{n}$. Using the coordinates $x^{1}, \cdots, x^{n}$ inherited from $\mathfrak{B}_{n}$, we find, by a direct calculation, that the only affine connection over $\mathfrak{M}_{n}$ invariant under $P_{n}$ is given by $\Gamma_{j k}^{\mathfrak{f}}(x)=0$. This gives us the space of type 2 in our theorem.

CASE $\mathrm{II}_{2} . S_{m}=P_{n-1}$. Here we shall be a little brief and omit the tedious Lie algebra arguments. Taking account of the fact $\Psi\left(G_{p}\right)=\tau\left(G_{p}\right)=L$, we first show that $S=P_{n-1}, R_{1}$ belongs to the radical $R$ of $G$ and that $G_{p}$ is reduc- 
tive $\left(^{(}\right)$in $G$. Then, by rather elaborate Lie algebra arguments, we can prove that $G$ has a basis

$$
e_{2}^{2}-e_{n}^{n}, e_{3}^{3}-e_{n}^{n}, \cdots, e_{n-1}^{n-1}, e_{\beta}^{\alpha}, e_{\alpha}^{1}, e_{0}^{1}, e_{0}^{\alpha} \quad(\alpha \neq \beta ; \alpha, \beta=2,3, \cdots, n)
$$

with the multiplication rule

$$
\left[e_{s}^{t}, e_{v}^{u}\right]=\delta_{s}^{u} e_{v}^{t}-\delta_{v}^{t} e_{s}^{u} \quad(s, t, u, v=0,1,2, \cdots, n)
$$

such that $G_{p}$ is spanned by

$$
e_{2}^{2}-e_{n}^{n}, \cdots, e_{n-1}^{n-1}, e_{\beta}^{\alpha}, e_{\alpha}^{1} \quad(\alpha \neq \beta) .
$$

Now let us consider the group $G^{\prime}$ of all transformations of $\mathfrak{B}_{n}$ of the form

$$
y^{1}=x^{1}+C_{\alpha}^{1} x^{\alpha}+C_{0}^{1}, \quad y^{\alpha}=C_{\beta}^{\alpha} x^{\beta}+C_{0}^{\alpha}, \quad\left|C_{\beta}^{\alpha}\right|=1 .
$$

This group $G^{\prime}$ is transitive over $\mathfrak{B}_{n}$ whose isotropic subgroup $G_{0}^{\prime}$ at the origin consists of transformations:

$$
y^{1}=x^{1}+C_{\alpha}^{1} x^{\alpha}, \quad y^{\alpha}=C_{\beta}^{\alpha} x^{\beta} .
$$

We see at once that, up to an isomorphism, $\left(G, G_{p}\right)=\left(G^{\prime}, G_{0}^{\prime}\right)$. By Remark 2 in $\S 11$, we can regard $\mathfrak{A}_{n}=\mathfrak{B}_{n}, G=G^{\prime}$. A direct calculation shows that the only affine connection over $\mathfrak{B}_{n}$ invariant under $G^{\prime}$ is given by $\Gamma_{j k}^{\mathfrak{l}}(x)=0$. This is the space of type 1 .

III. $\tau\left(G_{p}\right)=A_{p}=L^{\prime}$. In this case, we first show that $P_{n-1}$ is at the same time a maximal semi-simple subalgebra of $G$, and then we can show that the pair $\left(G, G_{p}\right)$ has only the following three possibilities:

CASE III I $_{1} G$ has a basis

$$
\begin{aligned}
e_{2}^{2}-e_{n}^{n}, \cdots, e_{n-1}^{n-1}-e_{n}^{n}, e_{\beta}^{\alpha}, e_{0}^{\alpha}, e_{1}^{\alpha}, \lambda e_{\alpha}^{\alpha}-e_{0}^{1}, \\
\alpha \neq \beta ; \alpha, \beta=2,3, \cdots, n ; \lambda \neq 0
\end{aligned}
$$

such that $G_{p}$ is spanned by

$$
e_{2}^{2}-e_{n}^{n}, \cdots, e_{n-1}^{n-1}-e_{n}^{n}, e_{\beta}^{\alpha}, e_{1}^{\alpha} \quad(\alpha \neq \beta) .
$$

In this case, we can regard $\mathfrak{A}_{n}=\mathfrak{B}_{n}$ and regard $G$ to be the group of all transformations of the form:

$$
y^{1}=x^{1}-t, \quad y^{\alpha}=C_{0}^{\alpha}+C_{1}^{\alpha} x^{1}+C_{\beta}^{\alpha} x^{\beta}, \quad\left|C_{\beta}^{\alpha}\right|=\exp (n-1) \lambda t .
$$

It follows then that the affine connections over $\mathfrak{B}_{n}$ invariant under $G$ must be of the form:

(8) A subalgebra $L$ of a Lie algebra $G$ is called reductive if there exists a linear subspace $R$ of $G$ such that $G=L+R, L \bigcap R \equiv 0,[L, R] \subset R$. 


$$
\Gamma_{j m}^{i}=k\left(\delta_{j}^{i} \delta_{m}^{1}+\delta_{m}^{i} \delta_{j}^{1}\right), \quad k=\text { constant. }
$$

When $k=0$, the space is of type 1 . When $k \neq 0$, we find that two connections corresponding to different $k$ 's are affinely equivalent (in the global sense). Thus we can assume $k=1$, and obtain the space of type 3 .

CASE III $_{2}$. $G$ and $G_{p}$ are spanned, respectively, by

$$
e_{2}^{2}-e_{n}^{n}, \cdots, e_{n-1}^{n-1}-e_{n}^{n}, e_{\beta}^{\alpha}, e_{1}^{\alpha}, e_{0}^{\alpha}, \quad \lambda\left(e_{0}^{0}+e_{1}^{1}\right)+e_{0}^{1}+e_{1}^{0}, \quad \alpha \neq \beta
$$

and

$$
e_{2}^{2}-e_{n}^{n}, \cdots, e_{n-1}^{n-1}-e_{n}^{n}, e_{\beta}^{\alpha}, e_{1}^{\alpha} .
$$

We can regard $\mathfrak{A}_{n}$ to be $\mathfrak{B}_{n}$, and $G$ to be the group of all transformations of the form

$y^{1}=x^{1}-t, \quad y^{\alpha}=C_{0}^{\alpha} \cosh x^{1}+C_{1}^{\alpha} \sinh x^{1}+C_{\beta}^{\alpha} x^{\beta}, \quad\left|C_{\beta}^{\alpha}\right|=\exp (n-1) \lambda t$.

The invariant affine connections are given by

$$
\begin{gathered}
\Gamma_{11}^{1}(x)=2 k, \quad \Gamma_{1 \beta}^{\alpha}(x)=k \delta_{\beta}^{\alpha}, \quad \Gamma_{11}^{\alpha}(x)=-x^{\alpha}, \quad \text { other } \Gamma=0, \\
k=\text { constant. }
\end{gathered}
$$

But the affine connections corresponding to $k$ and $-k$ are equivalent. Thus we can assume $k \geqq 0$, and get the spaces of type 4 .

CASE $\mathrm{III}_{3}$. $G$ and $G_{p}$ are spanned, respectively, by

$$
e_{2}^{2}-e_{n}^{n}, \cdots, e_{n-1}^{n-1}-e_{n}^{n}, e_{\beta}^{\alpha}, e_{1}^{\alpha}, e_{0}^{\alpha}, \quad \lambda\left(e_{0}^{0}+e_{1}^{1}\right)-e_{1}^{0}+e_{0}^{1}, \quad \alpha \neq \beta
$$

and

$$
e_{2}^{2}-e_{n}^{n}, \cdots, e_{n-1}^{n-1}-e_{n}^{n}, e_{\beta}^{\alpha}, e_{1}^{\alpha} .
$$

We can regard $\mathfrak{A}_{n}$ to be $\mathfrak{B}_{n}$ and $G$ to be the group of transformations of the form

$$
y^{1}=x^{1}-t, \quad y^{\alpha}=C_{0}^{\alpha} \cos x^{1}+C_{1}^{\alpha} \sin x^{1}+C_{\beta}^{\alpha} x^{\beta}, \quad\left|C_{\beta}^{\alpha}\right|=\exp (n-1) \lambda t .
$$

The invariant connections are given by

$$
\Gamma_{11}^{1}(x)=2 k, \quad \Gamma_{1 \beta}^{\alpha}(x)=k \delta_{\beta}^{\alpha}, \quad \Gamma_{11}^{\alpha}(x)=x^{\alpha}, \quad \text { other } \Gamma=0, \quad k \text { constant. }
$$

Just as in the above case, the connections corresponding to $k$ and $-k$ are equivalent. Thus we can assume $k \geqq 0$, and obtain the spaces of type 5 .

Thus we know that each $\mathfrak{A}_{n}$ satisfying the restrictions in Theorem 3 is equivalent to one of the five types. The completeness, curvature tensor and the maximal group of affine collineations of these five types of spaces can be obtained by a direct computation. 


\section{BIBLIOGRAPHY}

1. E. Cartan, Les groupes projectifs continus réls qui ne laissent invariantes accune multiplicite plane, J. Math. Pures Appl. vol. 10 (1914) pp. 149-186.

2. - Sur certaines formes riemanniennes remarquables des géometries a groupes fondamentales simples, Ann. Ecole Norm. vol. 44 (1927) pp. 345-567. 1933.

3. - Sur la structure des groupes de transformations finis et continus, 2d ed., Vuibert,

4. I. P. Egorov, On the order of the group of motions of spaces with affine connection, C. R. (Doklady) Acad. Sci. URSS vol. 57 (1947) pp. 867-870.

5. - On the groups of motions of spaces with asymmetric affine connection, ibid. vol. 64 (1949) pp. 621-624.

6. - Collineations of projectively connected spaces, ibid. vol. 80 (1951) pp. 709-712.

7. - A tensor characterization of $A_{n}$ of nonzero curvature with maximum mobility, ibid. vol. 84 (1952) pp. 209-212.

8. - Maximally mobile $L_{n}$ with a semi-symmetric connection, ibid. vol. 84 (1952) pp. $433-435$.

9. C. Ehresmann, Les connexions infinitesimales dans un espace fibré differentiable, Colloq. de Topologie, Bruxelles, 1950, pp. 29-55.

10. G. Fubini, Sugli spazii che ammettono un gruppo continuo di movimenti, Annali di Matematica Pura ed Applicata (3) vol. 8 (1903) pp. 39-81.

11. J. Levine, Classification of collineations in projectively and affinely connected spaces of two dimensions, Ann. of Math. vol. 52 (1950) pp. 465-477.

12. D. Montgomery and H. Samelson, Transformation groups of spheres, Ann. of Math. vol. 44 (1943) pp. 454-570.

13. Y. Muto, On the affinely connected spaces admitting a group of affine motions, Proc. Imp. Acad. Tokyo vol. 26 (1950) pp. 107-110.

14. K. Nomizu, On the group of affine transformations of an affinely connected manifold, Proc. Amer. Math. Soc. vol. 6 (1953) pp. 816-823.

15. K. Yano, Groups of transformations in generalized spaces, Akademia Press, Tokyo, 1949.

16. - On n-dimensional Riemannian spaces admitting a group of motions of order $n(n-1) / 2+1$, Trans. Amer. Math. Soc. vol. 74 (1953) pp. 260-279.

\footnotetext{
Alabama Polytechnic Institute, Auburn, Ala.

TOKYO UNIVERSITY, TOKYO, JAPAN.
} 